\title{
Effects of frequency detuning and excitation quantum number on the dynamics of entanglement in the Jaynes-Cummings polariton model
}

Christopher Mayero ( $\nabla$ cmayero@yahoo.com )

Maseno University, Kenya. https://orcid.org/0000-0002-8720-4211

\section{Patrick Owiny}

Maseno University, Kenya.

\section{Research Article}

Keywords: Jaynes-Cummings, Rabi oscillations, entanglement, concurrence

Posted Date: July 19th, 2021

DOl: https://doi.org/10.21203/rs.3.rs-727169/v1

License: (c) (1) This work is licensed under a Creative Commons Attribution 4.0 International License.

Read Full License 


\title{
Effects of frequency detuning and excitation quantum number on the dynamics of entanglement in the Jaynes-Cummings polariton model
}

\author{
Christopher Mayero ${ }^{1, *}$ and Patrick Owiny ${ }^{1}$ \\ ${ }^{1}$ Maseno University, Department of Physics and Materials Science, Private Bag-40105, Maseno, Kenya.
}

(Dated: April 1, 2021)

\begin{abstract}
We present a scheme for generating polaritons which are maximally entangled qubit states in the Jaynes-Cummings interaction mechanism. Considering a specific case of an atom initially in an excited state entering a cavity mode initially in vacuum state and in a non-resonant atomfield Jaynes-Cummings interaction, we demonstrate using graphical representation on the Bloch sphere that an increase in frequency detuning leads to an increase in Rabi oscillations. Analysis of the dynamical behaviour of quantum entanglement in the general Jaynes-Cummings atom-field interactions measured by concurrence show that frequency detuning and photon number parameters are vital in enhancing entanglement.
\end{abstract}

Keywords: Jaynes-Cummings, Rabi oscillations, entanglement, concurrence

\section{INTRODUCTION}

We begin by giving a general observation that the Jaynes-Cummings model (JC) [1] originally presented in a 1963 article by Edwin Jaynes and Fred Cummings made clear the idea of a fully quantum mechanical interaction between a two-level atom and a quantized single mode electromagnetic field. The JC model aims to find how quantization of the radiation field affects the predictions for the evolution of the state of a two-level system, in comparison with semi-classical theory of matterradiation interaction $[2,3]$. What is a purely quantum effect described only by the JC model and not the semiclassical theory, is the revival of the atomic population inversion after its collapse, providing a direct evidence of the discreteness of the field states (photons).

In order to precisely describe the interaction between an atom and a laser field, the JC model is generalized in different ways. Some of the generalizations comprehend different initial conditions [4-7] dissipation and damping in the model [8], multilevel atoms and multiple atoms [9], and multi-mode description of the field [10]. Other extensions of the JC model include a driving pump laser acting on one or more two-level atoms, which allows simultaneous action of rotating and counter-rotating coupling terms in the Hamiltonian, including dissipative effects due to Markovian environments [11, 12]. Recently, it has been shown in [4-6] that the quantum Rabi model $(\mathrm{QRM})[4,13]$ is composed of a rotating component, dominated by an exactly solvable the JC interaction specified by a conserved JC excitation number operator which generates the U(1) symmetry of the rotating frame (RF) and a counter-rotating component, the anti-Jaynes-Cummings (AJC) interaction specified by a conserved AJC excitation number operator which generates the $\mathrm{U}(1)$ symmetry of the counter rotating frame (CRF). In the reformulation [4-6], consistent generalisation of the initial states to corresponding $n \geq 0$ entangled

\footnotetext{
* E-mail address: cmayero@yahoo.com
}

states of the JC in the RF (polariton) and AJC in the CRF (anti-polariton), provides general dynamical evolution of the QRM characterised by collapses and revivals of in the time-evolution of the atomic, field mode, JC and AJC excitation numbers for large initial photon numbers. The JC and AJC excitation numbers are conserved [4] in the respective $\mathrm{RF}$ and $\mathrm{CRF}$, but each evolve in time in the alternate frame. In this paper a specified analysis of dynamics of the JC polariton model posed in our initial work [14] as a comparison to the AJC interaction mechanism is presented. We are interested in analysis of quantum state configuration of the qubit states and entanglement of qubits in the JC polariton model $[5,6]$. To do this, we study the effect of frequency detuning and photon number on the dynamics of the JC polariton model.

The content of this paper is therefore summarized as follows. Section II presents an overview of the theoretical model. In section III, Rabi oscillations in the JC model is studied. Entanglement analysis of the JC qubit state vectors and time-evolution of entanglement of the general JC interaction is presented in section IV and finally section V contains the conclusion.

\section{THE MODEL}

The quantum Rabi model of a quantized electromagnetic field mode interacting with a two-level atom is generated by the Hamiltonian [4-6]

$$
\hat{H}_{R}=\frac{1}{2} \hbar \omega\left(\hat{a}^{\dagger} \hat{a}+\hat{a} \hat{a}^{\dagger}\right)+\hbar \omega_{0} \hat{s}_{z}+\hbar \lambda\left(\hat{a}+\hat{a}^{\dagger}\right)\left(\hat{s}_{+}+\hat{s}(\mathfrak{l})\right.
$$

noting that the free field mode Hamiltonian is expressed in normal and anti-normal order form $\frac{1}{2} \hbar \omega\left(\hat{a}^{\dagger} \hat{a}+\right.$ $\left.\hat{a} \hat{a}^{\dagger}\right)$. Here, $\omega, \hat{a}, \hat{a}^{\dagger}$ are quantized field mode angular frequency, annihilation and creation operators, while $\omega_{0}, \hat{s}_{z}, \hat{s}_{+}, \hat{s}_{-}$are atomic state transition angular frequency and operators. The Rabi Hamiltonian in eq. (1) is expressed in a symmetrized two-component form [4-6] 


$$
\hat{H}_{R}=\frac{1}{2}(\hat{H}+\hat{\bar{H}})
$$

where $\hat{H}$ is the standard JC Hamiltonian interpreted as a polariton qubit Hamiltonian expressed in the form [4-6]

$$
\begin{aligned}
& \hat{H}=\hbar \omega \hat{N}+2 \hbar \lambda \hat{A}-\frac{1}{2} \hbar \omega \quad ; \quad \hat{N}=\hat{a}^{\dagger} \hat{a}+\hat{s}_{+} \hat{s}_{-} \quad ; \\
& \hat{A}=\alpha \hat{s}_{z}+\hat{a} \hat{s}_{+}+\hat{a}^{\dagger} \hat{s}_{-} \quad ; \quad \alpha=\frac{\omega_{0}-\omega}{2 \lambda}
\end{aligned}
$$

In Eq. (3), $\hat{N}$ and $\hat{A}$ are the respective polariton qubit conserved excitation number and state transition operator.

Following the physical property established in [6], that for the field mode in an initial vacuum state only an atom in an initial excited state $|e\rangle$ entering the cavity couples to the rotating positive frequency field component in the JC interaction mechanism, we generally take the atom to be in an initial excited state $|e\rangle$ in the JC model.

Considering the JC dynamics, applying the state transition operator $\hat{A}$ from Eq. (3) to the initial atom-field $n$-photon excited state vector $|e, n\rangle$, the basic qubit state vectors $\left|\psi_{e n}\right\rangle$ and $\left|\phi_{e n}\right\rangle$ are determined in the form $(n=0,1,2, \ldots).[6]$

$$
\left|\psi_{e n}\right\rangle=|e, n\rangle \quad ; \quad\left|\phi_{e n}\right\rangle=c_{e n}|e, n\rangle+s_{e n}|g, n+1\rangle
$$

with dimensionless interaction parameters $c_{e n}, s_{e n}$ and Rabi frequency $R_{e n}$ defined as

$$
\begin{aligned}
c_{e n} & =\frac{\delta}{2 R_{e n}} ; \quad s_{e n}=\frac{2 \lambda \sqrt{n+1}}{R_{e n}} ; \quad R_{e n}=2 \lambda A_{e n} ; \\
A_{e n} & =\sqrt{(n+1)+\frac{\delta^{2}}{16 \lambda^{2}}} ; \delta=\omega_{0}-\omega
\end{aligned}
$$

where we have introduced detuning $\delta=\omega_{0}-\omega$ to redefine $\alpha$ in Eq. (3).

The qubit state vectors in Eq. (4) satisfy the qubit state transition algebraic operations

$$
\hat{A}\left|\psi_{e n}\right\rangle=A_{e n}\left|\phi_{e n}\right\rangle \quad ; \quad \hat{A}\left|\phi_{e n}\right\rangle=A_{e n}\left|\psi_{e n}\right\rangle
$$

In the JC qubit subspace spanned by normalized but nonorthogonal basic qubit state vectors $\left|\psi_{e n}\right\rangle,\left|\phi_{e n}\right\rangle$ the basic qubit state transition operator $\hat{\varepsilon}_{e}$ and identity operator $\hat{I}_{e}$ are introduced according to the definition

$$
\hat{\varepsilon}_{e}=\frac{\hat{A}}{A_{e n}} \quad ; \quad \hat{I}_{e}=\frac{\hat{A}^{2}}{A_{e n}^{2}} \quad \Rightarrow \quad \hat{\varepsilon}_{e}^{2}=\hat{I}_{e}
$$

which on substituting into Eq. (6) generates the basic qubit state transition algebraic operations

$$
\begin{aligned}
& \hat{\varepsilon}_{e}\left|\psi_{e n}\right\rangle=\left|\phi_{e n}\right\rangle \quad ; \quad \hat{\varepsilon}_{e}\left|\phi_{e n}\right\rangle=\left|\psi_{e n}\right\rangle \quad ; \\
& \hat{I}_{e}\left|\psi_{e n}\right\rangle=\left|\psi_{e n}\right\rangle \quad ; \quad \hat{I}_{e}\left|\phi_{e n}\right\rangle=\left|\phi_{e n}\right\rangle
\end{aligned}
$$

The algebraic properties $\hat{\varepsilon}_{e}^{2 k}=\hat{I}_{e}$ and $\hat{\varepsilon}_{e}^{2 k+1}=\hat{\varepsilon}_{e}$ easily gives the final property $(k=0,1,2, \ldots)$

$$
e^{i \theta \hat{\varepsilon}_{e}}=\cos (\theta) \hat{I}_{e}+i \sin (\theta) \hat{\varepsilon}_{e}
$$

which is useful in evaluating the time-evolution operator.

The JC qubit Hamiltonian defined within the qubit subspace spanned by the basic qubit state vector vectors $\left|\psi_{e n}\right\rangle,\left|\phi_{e n}\right\rangle$ is then expressed in terms of the basic qubit state transition operators $\hat{\varepsilon}_{e}, \hat{I}_{e}$ in the form [6]

$$
\hat{H}_{e}=\hbar \omega\left(n+\frac{1}{2}\right) \hat{I}_{e}+\hbar R_{e n} \hat{\varepsilon}_{e}
$$

We use this form of the JC Hamiltonian to determine the general time-evolving state vector describing Rabi oscillations in the JC dynamics in Sec. III.

\section{RABI OSCILLATIONS BETWEEN THE BASIC JC QUBIT STATE VECTORS $\left|\psi_{e n}\right\rangle$ AND $\left|\phi_{e n}\right\rangle$}

The general dynamics generated by the JC Hamiltonian in Eq. (10) is described by a time evolving JC qubit state vector $\left|\Psi_{e n}(t)\right\rangle$ obtained from the time-dependent Schrödinger equation in the form [6]

$$
\left|\Psi_{e n}(t)\right\rangle=\hat{U}_{e}(t)\left|\psi_{e n}\right\rangle \quad ; \quad \hat{U}_{e}(t)=e^{-\frac{i}{\hbar} \hat{H}_{e} t}
$$

where $\hat{U}_{e}(t)$ is the time evolution operator. Substituting $\hat{H}_{e}$ from Eq. (10) into Eq. (11) and applying appropriate algebraic properties [6], we use the relation in Eq. (9) to express the time evolution operator in its final form

$$
\hat{U}_{e}(t)=e^{-i \omega t\left(n+\frac{1}{2}\right)}\left\{\cos \left(R_{e n} t\right) \hat{I}_{e}-i \sin \left(R_{e n} t\right) \hat{\varepsilon}_{e}\right\}
$$

which we substitute into equation Eq. (11) and use the qubit state transition operations in Eq. (8) to obtain the time-evolving JC qubit state vector in the form

$$
\left|\Psi_{e n}(t)\right\rangle=e^{-i \omega t\left(n+\frac{1}{2}\right)}\left\{\cos \left(R_{e n} t\right)\left|\psi_{e n}\right\rangle-i \sin \left(R_{e n} t\right)\left|\phi_{e n}\right\rangle\right\}
$$

This time evolving state vector describes Rabi oscillations between the basic qubit states $\left|\psi_{e n}\right\rangle$ and $\left|\phi_{e n}\right\rangle$ at Rabi frequency $R_{e n}$.

In order to determine the length of the Bloch vector associated with the state vector in Eq. (13), we introduce the density operator

$$
\hat{\rho}_{e n}(t)=\left|\Psi_{e n}(t)\right\rangle\left\langle\Psi_{e n}(t)\right|
$$


which we expand to obtain

$$
\begin{aligned}
\hat{\rho}_{e n}(t) & =\cos ^{2}\left(R_{e n} t\right)\left|\psi_{e n}\right\rangle\left\langle\psi_{e n}\left|+\frac{i}{2} \sin \left(2 R_{e n} t\right)\right| \psi_{e n}\right\rangle\left\langle\phi_{e n}\right| \\
& -\frac{i}{2} \sin \left(2 R_{e n} t\right)\left|\phi_{e n}\right\rangle\left\langle\psi_{e n}\left|+\sin ^{2}\left(R_{e n} t\right)\right| \phi_{e n}\right\rangle\left\langle\phi_{e n}\right|
\end{aligned}
$$

Defining the coefficients of the projectors in Eq. (14b) as

$$
\begin{aligned}
& \rho_{e n}^{11}(t)=\cos ^{2}\left(R_{e n} t\right) \quad ; \quad \rho_{e n}^{12}(t)=\frac{i}{2} \sin \left(2 R_{e n} t\right) ; \\
& \rho_{e n}^{21}(t)=-\frac{i}{2} \sin \left(2 R_{e n} t\right) \quad ; \quad \rho_{e n}^{22}(t)=\sin ^{2}\left(R_{e n} t\right)
\end{aligned}
$$

and interpreting the coefficients in Eq. (14c) as elements of a $2 \times 2$ density matrix $\rho_{e n}(t)$, which we express in terms of standard Pauli operator matrices $I, \sigma_{x}, \sigma_{y}$ and $\sigma_{z}$ as

$$
\rho_{e n}(t)=\left(\begin{array}{cc}
\rho_{e n}^{11}(t) & \rho_{e n}^{12}(t) \\
\rho_{e n}^{21}(t) & \rho_{e n}^{22}(t)
\end{array}\right)=\frac{1}{2}\left(I+\vec{\rho}_{e n}(t) \cdot \vec{\sigma}\right)
$$

where $\vec{\sigma}=\left(\sigma_{x}, \sigma_{y}, \sigma_{z}\right)$ is the Pauli matrix vector and we have introduced the time-evolving Bloch vector $\vec{\rho}_{e n}(t)$ obtained in the form

$$
\vec{\rho}_{e n}(t)=\left(\rho_{e n}^{x}(t), \rho_{e n}^{y}(t), \rho_{e n}^{z}(t)\right)
$$

with components defined as

$$
\begin{aligned}
& \rho_{e n}^{x}(t)=\rho_{e n}^{12}(t)+\rho_{e n}^{21}(t)=0 ; \\
& \rho_{e n}^{y}(t)=i\left(\rho_{e n}^{12}(t)-\rho_{e n}^{21}(t)\right)=-\sin \left(2 \bar{R}_{e n} t\right) ; \\
& \rho_{e n}^{z}(t)=\rho_{e n}^{11}(t)-\rho_{e n}^{22}(t)=\cos \left(2 \bar{R}_{e n} t\right)
\end{aligned} ;
$$

The Bloch vector in Eq. (14e) takes the explicit form

$$
\vec{\rho}_{e n}(t)=\left(0,-\sin \left(2 R_{e n} t\right), \cos \left(2 R_{e n} t\right)\right)
$$

which has unit length obtained easily as

$$
\left|\vec{\rho}_{e n}(t)\right|=1
$$

The property that the Bloch vector $\vec{\rho}_{e n}(t)$ is of unit length (the Bloch sphere has unit radius), clearly shows that the general time evolving state vector $\left|\Psi_{e n}(t)\right\rangle$ in Eq. (13) is a pure state.

We now proceed to demonstrate the time evolution of the Bloch vector $\vec{\rho}_{\text {en }}(t)$ which in effect describes the geometric configuration of states. We have adopted class 4 Bloch-sphere entanglement of a quantum rank- 2 bipartite state $[15,16]$ to bring a clear visualization of this interaction. In this respect, we consider the specific example (which also applies to the general $n$-photon case) of an atom initially in excited state $|e\rangle$ entering a cavity with the field mode starting off in an initial vacuum state $|0\rangle$, such that the initial atom-field state is $|e, 0\rangle$.

In the specific example starting with an atom in the excited state $|e\rangle$ and the field mode in the vacuum state $|0\rangle$ the basic qubit state vectors $\left|\psi_{e 0}\right\rangle$ and $\left|\phi_{e 0}\right\rangle$, together with the corresponding entanglement parameters, are obtained by setting $n=0$ in Eqs. (4) and (5) in the form

$$
\begin{gathered}
\left|\psi_{e 0}\right\rangle=|e, 0\rangle \quad ; \quad\left|\phi_{e o}\right\rangle=c_{e 0}|e, 0\rangle+s_{e 0}|g, 1\rangle \\
c_{e 0}=\frac{\delta}{2 R_{e 0}} \quad ; \quad s_{e 0}=\frac{2 \lambda}{R_{e 0}} \quad ; \quad R_{e 0}=\frac{1}{2} \sqrt{16 \lambda^{2}+\delta^{2}} \\
|e, 0\rangle=|e\rangle \otimes|0\rangle \quad ; \quad|g, 1\rangle=|g\rangle \otimes|1\rangle
\end{gathered}
$$

The corresponding Hamiltonian in Eq. (10) becomes $(n=$ $0)$

$$
\hat{H}_{e}=\frac{1}{2} \hbar \omega \hat{I}_{e}+\hbar R_{e 0} \hat{\varepsilon}_{e}
$$

The time-evolving state vector in Eq. (13) takes the form $(n=0)$

$$
\left|\Psi_{e 0}(t)\right\rangle=e^{-\frac{i \omega t}{2}}\left\{\cos \left(R_{e 0} t\right)\left|\psi_{e 0}\right\rangle-i \sin \left(R_{e 0} t\right)\left|\phi_{e 0}\right\rangle\right\}
$$

which describes Rabi oscillations at frequency $R_{e 0}$ between the initial separable qubit state vector $\left|\psi_{e 0}\right\rangle$ and the entangled qubit state vector $\left|\phi_{e 0}\right\rangle$.

The Rabi oscillation process is best described by the corresponding Bloch vector which follows from Eq. (14g) in the form $(n=0)$

$$
\vec{\rho}_{e 0}(t)=\left(0,-\sin \left(2 R_{e 0} t\right), \cos \left(2 R_{e 0} t\right)\right)
$$

The time evolution of this Bloch vector reveals that the Rabi oscillations between the basic qubit state vectors $\left|\psi_{e 0}\right\rangle,\left|\phi_{e 0}\right\rangle$ describe circles on which the states are distributed on the Bloch sphere as we demonstrate in Fig. 1 below.

In Fig. 1 we have plotted the JC Rabi oscillation process with respective Rabi frequencies $R_{e 0}$ determined according to Eq. (15) for arbitrary values of detuning $\delta=\omega_{0}-\omega$.

In the present work, for various values of $\delta=$ $\lambda, 2 \lambda, 3 \lambda, 4 \lambda, 0$ and field mode frequency $\omega=2 \lambda$ we use the general time evolving state vector in Eq. (17), with $R_{e 0}$ as defined in Eq. (15) to determine the coupled qubit state vectors $\left|\psi_{e 0}\right\rangle,\left|\phi_{e 0}\right\rangle$ in Eq. (15) by setting $R_{e 0} t=\frac{\pi}{2}$, describing half cycle of Rabi oscillation as presented below. In each case we have an accumulated global phase factor which does not affect measurement results [17-19], but we have maintained them here in Eqs. (19a) - (19e) to explain the continuous time evolution over one cycle. $\delta=0$ :

$$
|e, 0\rangle \rightarrow e^{-i \pi \frac{3}{4}}|g, 1\rangle \rightarrow e^{-i \pi \frac{3}{2}}|e, 0\rangle
$$

$\delta=\lambda$ :

$$
|e, 0\rangle \rightarrow e^{-i \pi \frac{25}{34}}\left\{\frac{1}{\sqrt{17}}|e, 0\rangle+\frac{4}{\sqrt{17}}|g, 1\rangle\right\} \rightarrow e^{-i \pi \frac{25}{17}}|e, 0\rangle
$$




$$
\begin{aligned}
& \delta=2 \lambda: \\
& |e, 0\rangle \rightarrow e^{-i \pi \frac{7}{10}}\left\{\frac{1}{\sqrt{5}}|e, 0\rangle+\frac{2}{\sqrt{5}}|g, 1\rangle\right\} \rightarrow e^{-i \pi \frac{14}{10}}|e, 0\rangle
\end{aligned}
$$

$\delta=3 \lambda$

$$
|e, 0\rangle \rightarrow e^{-i \pi \frac{7}{10}}\left\{\frac{3}{5}|e, 0\rangle+\frac{4}{5}|g, 1\rangle\right\} \rightarrow e^{-i \pi \frac{14}{10}}|e, 0\rangle
$$

$\delta=4 \lambda$

$$
|e, 0\rangle \rightarrow e^{-i \pi \frac{7}{10}}\left\{\frac{1}{\sqrt{2}}|e, 0\rangle+\frac{1}{\sqrt{2}}|g, 1\rangle\right\} \rightarrow e^{-i \pi \frac{14}{10}}|e, 0\rangle
$$

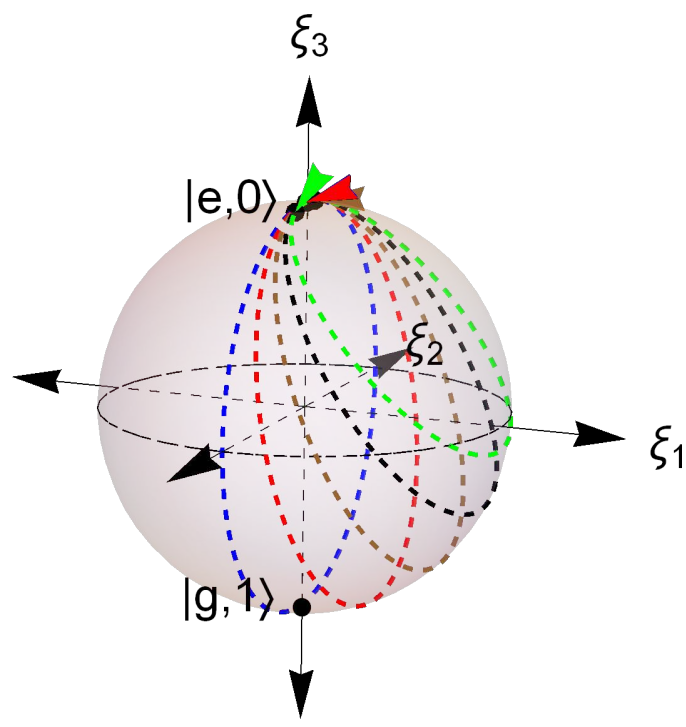

FIG. 1: Rabi oscillations in JC interaction mechanism. Here, blue circle is at resonance with detuning $\delta=\omega_{0}-$ $\omega=0$, red circle is for detuning $\delta=\lambda$, brown $\delta=2 \lambda$, black circle for detuning $\delta=3 \lambda$ and green $\delta=4 \lambda$.

The JC Rabi oscillations for cases $\delta=\lambda, 2 \lambda, 3 \lambda, 4 \lambda, 0$ are plotted as red, brown, black, green and blue circles in Fig. 1. Here, Fig. 1 is a Bloch sphere entanglement [15] that corresponds to a 2-dimensional subspace of $\mathbb{C}^{2} \otimes$ $\mathbb{C}^{2} \operatorname{Span}\left\{|e, 0\rangle, c_{e 0}|e, 0\rangle+s_{e 0}|g, 1\rangle\right\}$ with $c_{e 0}=\frac{\delta}{2 R_{e 0}}$ and $s_{e 0}=\frac{2 \lambda}{R_{e 0}}$, where we recall that, in the JC interaction the initial atom-field ground state with the field mode in the vacuum state is $|e, 0\rangle$.

We clearly see in Fig. 1 that in a resonant atom-field interaction $\delta=0$, Rabi oscillations occur between $|e, 0\rangle$ and $|g, 1\rangle$ and the Bloch vector in Eq. (18) describes a path along the $y z$-plane as shown by the blue circle, while in a non-resonant $\delta \neq 0$ atom-field interaction, effective Rabi state transition oscillations occur only between $|e, 0\rangle$ and a linear superposition of $|e, 0\rangle$ and $|g, 1\rangle$ with time evolution following a circular path described by the Bloch vector in Eq. (18) at an axis about the North pole of the Bloch sphere (see the red, brown, black and green circles as examples). It is also clear in Fig. 1 that an increase in the frequency detuning parameter in a non-resonant atom-field interaction $\delta \neq 0$ results into an increase in Rabi frequency and hence a reduction in the period of Rabi oscillations as demonstrated by reduction in size of the Rabi oscillation circles (red, brown, black and green).

The geometric configuration of the state space demonstrated on the Bloch-sphere in Fig. 1 determined using the approach in [6] agrees precisely with that determined using the semi-classical approach in [20] corresponding to a 2-dimensional subspace of $\mathbb{C}^{2}$ Span $\{|e\rangle,|g\rangle\}$. In the approach [20], at resonance where detuning $\delta=0$ the atomic population is inverted from $|e\rangle$ to $|g\rangle$ and the Bloch-vector $\vec{r}=(\sin (\theta) \cos (\phi), \sin (\theta) \sin (\phi), \cos (\theta))$ describes a path along the $y z$-plane on the Bloch-sphere. For other values of detuning, the atom evolves from $|e\rangle$ to a linear superposition of $|e\rangle$ and $|g\rangle$ and back to $|e\rangle$ and the Bloch-vector $\vec{r}$ describes a circle about the North pole of the Bloch-sphere.

\section{ENTANGLEMENT PROPERTIES}

In quantum information, it is of interest to measure or quantify the entanglement of states. In a bipartite case, with two sub-systems A, B concurrence [17, 21, 22] is a good measure of entanglement in practical situations. For pure states of two spins, concurrence is defined as

$$
C\left(\left|\psi_{A B}\right\rangle\right)=\left|\left\langle\psi_{A B} \mid \tilde{\psi}_{A B}\right\rangle\right|
$$

where $\left|\tilde{\psi}_{A B}\right\rangle \equiv \hat{\sigma}_{y}^{\otimes 2}\left|\psi_{A B}^{*}\right\rangle$ is referred to as the 'spinflipped' state vector [21]. Further, concurrence can be expressed in terms of the minimum average pure-state concurrence where the required minimum is to be taken over all possible ways of decomposing the ensemble $\hat{\rho}_{A B}$ into a mixture of pure states $\left|\psi_{A B}\right\rangle$. In this respect, for a general two-spin state, concurrence is defined as

$$
\begin{aligned}
C\left(\rho_{A B}\right) & =\max \left\{0, \lambda_{1},-\lambda_{2},-\lambda_{3},-\lambda_{4}\right\} \quad ; \\
\hat{\rho}_{A B} & =\left|\psi_{A B}\right\rangle\left\langle\psi_{A B}\right| \quad ; \quad \hat{\tilde{\rho}}_{A B}=\hat{\rho}_{A B} \hat{\sigma}_{y}^{\otimes 2} \hat{\rho}_{A B}^{*} \hat{\sigma}_{y}^{\otimes 2}
\end{aligned}
$$

where $\lambda_{i}$ are real square-roots of the eigenvalues of the matrix $\hat{\tilde{\rho}}_{A B}$ in Eq. (21). Concurrence varies from 0 for a completely disentangled state to 1 for a maximally entangled state.

In this section we analyse the entanglement properties of the qubit state vectors and the dynamical evolution of entanglement generated in the JC interaction. 


\section{A. Entanglement analysis of basic qubit state vectors $\left|\psi_{e 0}\right\rangle$ and $\left|\phi_{e 0}\right\rangle$}

Let us start by considering the entanglement properties of the initial state $\left|\psi_{e 0}\right\rangle$ which according to the definition in Eq. (15) is a separable pure state. In this context

$$
\left|\tilde{\psi}_{e 0}\right\rangle=\hat{\sigma}_{y}^{\otimes 2}\left|\psi_{e 0}^{*}\right\rangle=\hat{\sigma}_{y}^{\otimes 2}(|e\rangle \otimes|0\rangle)=i|g\rangle \otimes i|1\rangle
$$

substituting into Eq. (20) we obtain

$$
C\left(\left|\psi_{e 0}\right\rangle\right)=\left|\left\langle\psi_{e 0} \mid \tilde{\psi}_{e 0}\right\rangle\right|=|-\langle e \mid g\rangle\langle 0 \mid 1\rangle|=0
$$

quantifying the state in Eq. (15) (or the initial qubit state in Eq. (19a)) as a pure product state $|e\rangle \otimes|0\rangle$. Similarly, ignoring the global phase factor in Eq. (19a), the transition qubit state $\left|\phi_{e 0}\right\rangle=|g, 1\rangle$ obtained at resonance $\delta=0$ is a pure product state $|g\rangle \otimes|1\rangle$.

We now proceed to determine the entanglement property of the transition qubit state vector $\left|\phi_{e 0}\right\rangle$ obtained at detuning $\delta=\lambda$ in Eq. (19b). Ignoring the global phase factor in Eq. (19b), the transition qubit state $\left|\phi_{e 0}\right\rangle$ takes the form

$$
\delta=\lambda \quad: \quad \frac{1}{\sqrt{17}}|e, 0\rangle+\frac{4}{\sqrt{17}}|g, 1\rangle
$$

The corresponding density operator of the state in Eq. (24) is

$$
\begin{aligned}
\hat{\rho}_{e 0} & =\left|\phi_{e 0}\right\rangle\left\langle\phi_{e 0}\right| \\
& =\frac{1}{17}|e, 0\rangle\left\langle e, 0\left|+\frac{4}{17}\right| e, 0\right\rangle\left\langle g, 1\left|+\frac{4}{17}\right| g, 1\right\rangle\langle e, 0| \\
& +\frac{16}{17}|g, 1\rangle\langle g, 1|
\end{aligned}
$$

which takes the explicit $4 \times 4$ matrix form

$$
\hat{\rho}_{e 0}=\frac{1}{17}\left(\begin{array}{cccc}
1 & 0 & 0 & 4 \\
0 & 0 & 0 & 0 \\
0 & 0 & 0 & 0 \\
4 & 0 & 0 & 16
\end{array}\right)
$$

and $\hat{\tilde{\rho}}_{e 0}$ by applying the definition in Eq. (21) is

$$
\hat{\tilde{\rho}}_{e 0}=\hat{\rho}_{e 0}\left(\hat{\sigma}_{y}^{\otimes 2}\right) \hat{\rho}_{e 0}^{*}\left(\hat{\sigma}_{y}^{\otimes 2}\right)
$$

which takes the explicit $4 \times 4$ matrix form

$$
\hat{\tilde{\rho}}_{e 0}=\frac{1}{289}\left(\begin{array}{cccc}
32 & 0 & 0 & 8 \\
0 & 0 & 0 & 0 \\
0 & 0 & 0 & 0 \\
128 & 0 & 0 & 32
\end{array}\right)
$$

The eigenvalues of the matrix in Eq (28) are

$$
\lambda_{1}=\frac{64}{289}, \lambda_{2}=0, \lambda_{3}=0, \lambda_{4}=0
$$

Substituting the eigenvalues in Eq. (29) into Eq. (21) we obtain

$$
C\left(\hat{\rho}_{e 0}\right)=\max \left\{0, \sqrt{\frac{64}{289}}\right\}=\frac{8}{17}<1
$$

quantifying the transition qubit state in Eq. (24) (or Eq. (19b)) as an entangled state but not maximally entangled since $C\left(\hat{\rho}_{e 0}\right)<1$. Similarly, the transition qubit states $\left|\phi_{e 0}\right\rangle=\frac{1}{\sqrt{5}}|e, 0\rangle+\frac{2}{\sqrt{5}}|g, 1\rangle$ obtained for detuning $\delta=2 \lambda$ in Eq. (19c) and $\left|\phi_{e 0}\right\rangle=\frac{3}{5}|e, 0\rangle+\frac{4}{5}|g, 1\rangle$ obtained for detuning $\delta=3 \lambda$ in Eq. (19d) are entangled but not maximally entangled.

Finally we consider the case of detuning $\delta=4 \lambda$. Once again ignoring the global phase factor in Eq. (19e), the transition qubit state vector takes the form

$$
\delta=4 \lambda \quad: \quad \frac{1}{\sqrt{2}}|e, 0\rangle+\frac{1}{\sqrt{2}}|g, 1\rangle
$$

The corresponding density operator of the state in Eq. (31) is

$$
\begin{aligned}
\hat{\rho}_{e 0} & =\frac{1}{2}|e, 0\rangle\left\langle e, 0\left|+\frac{1}{2}\right| e, 0\right\rangle\left\langle g, 1\left|+\frac{1}{2}\right| g, 1\right\rangle\langle e, 0| \\
& +\frac{1}{2}|g, 1\rangle\langle g, 1|
\end{aligned}
$$

The explicit $4 \times 4$ matrix forms of $\hat{\rho}_{e 0}$ in Eq. (32) and $\hat{\tilde{\rho}}_{e 0}$ by applying the definition in Eq. (21) are of the form

$$
\hat{\rho}_{e 0}=\frac{1}{2}\left(\begin{array}{cccc}
1 & 0 & 0 & 1 \\
0 & 0 & 0 & 0 \\
0 & 0 & 0 & 0 \\
1 & 0 & 0 & 1
\end{array}\right) \quad ; \quad \hat{\tilde{\rho}}_{e 0}=\frac{1}{2}\left(\begin{array}{llll}
1 & 0 & 0 & 1 \\
0 & 0 & 0 & 0 \\
0 & 0 & 0 & 0 \\
1 & 0 & 0 & 1
\end{array}\right)
$$

The eigenvalues of $\hat{\tilde{\rho}}_{e 0}$ in Eq. (33) are

$$
\lambda_{1}=1, \lambda_{2}=0, \lambda_{3}=0, \lambda_{4}=0
$$

which on substituting into Eq. (21) we obtain

$$
C\left(\hat{\tilde{\rho}}_{e 0}\right)=\max \{0,1\}=1
$$

The unit concurrence determined in Eq. (35) reveals that the transition qubit state vector in Eq. (31) (or Eq. (19e) determined at detuning $\delta=4 \lambda$ is a maximally entangled bipartite pure state.

A similar proof of entanglement of the JC qubit states is easily achieved for all possible values of frequency detuning parameter $\delta=\omega_{0}-\omega$, confirming that in the initial vacuum-field JC interaction, when $\delta \neq 0$ reversible 
transitions occur only between a pure initial separable qubit state vector and a pure entangled qubit state vector while when $\delta=0$ reversible transitions occur only between pure initial and transition separable qubit state vectors. These properties of Rabi oscillations occurs in the general JC interaction described by the general time evolving state vector $\left|\Psi_{e n}(t)\right\rangle$ in Eq. (13).

\section{B. Entanglement evolution}

Let us consider the general dynamics of the JC interaction described by the general time-evolving qubit state vector $\left|\Psi_{e n}(t)\right\rangle$ in Eq. (13). Substituting $\left|\Psi_{e n}(t)\right\rangle$ from Eq. (13) into Eq. (14a) and using the definitions of $\left|\psi_{e n}\right\rangle$ and $\left|\phi_{e n}\right\rangle$ in Eq. (4) the density operator takes the form

$$
\begin{aligned}
\hat{\rho}_{e n}(t) & =\left\{\cos ^{2}\left(R_{e n} t\right)+c_{e n}^{2} \sin ^{2}\left(R_{e n} t\right)\right\}|e, n\rangle\langle e, n| \\
& +\left\{i s_{e n} \cos \left(R_{e n} t\right) \sin \left(R_{e n} t\right)\right. \\
& \left.+c_{e n} s_{e n} \sin ^{2}\left(R_{e n} t\right)\right\}|e, n\rangle\langle g, n+1| \\
& +\left\{-i s_{e n} \cos \left(R_{e n} t\right) \sin \left(R_{e n} t\right)\right. \\
& \left.+c_{e n} s_{e n} \sin ^{2}\left(R_{e n} t\right)\right\}|g, n+1\rangle\langle e, n| \\
& +\left\{s_{e n}^{2} \sin ^{2}\left(R_{e n} t\right)\right\}|g, n+1\rangle\langle g, n+1|
\end{aligned}
$$

The reduced density operator of the atom is determined by tracing over the field states thus taking the form

$$
\hat{\rho}_{A}(t)=\operatorname{tr}_{F}\left(\hat{\rho}_{e n}(t)\right)=P_{e}(t)|e\rangle\left\langle e\left|+P_{g}(t)\right| g\right\rangle\langle g|
$$

after introducing the general time-evolving atomic state probabilities $P_{e}(t)$ and $P_{g}(t)$ obtained as

$$
\begin{aligned}
& P_{e}(t)=\cos ^{2}\left(R_{e n} t\right)+c_{e n}^{2} \sin ^{2}\left(R_{e n} t\right) ; \\
& P_{g}(t)=s_{e n}^{2} \sin ^{2}\left(R_{e n} t\right)
\end{aligned}
$$

The degree of entanglement (DEM) using concurrence measure which was formulated as a convex measure to amount the DEM for two qubits in pure states by Wooters and Hill [23] is defined as

$$
C(t)=\sqrt{2\left(1-\operatorname{tr} \hat{\rho}^{2}\right)}
$$

where $\hat{\rho}$ is the reduced density operator. Substituting $\hat{\rho}_{A}(t)$ in Eq. (36b) into Eq. (37) and using the standard definitions of $c_{e n}, s_{e n}$ and $R_{e n}$ in Eq. (5) to evaluate the probabilities in Eq. (36c), we plot concurrence $C(\tau)$ Eq. (37) against scaled time $\tau=\lambda t$ at resonance where the frequency detuning $\delta=0$, arbitrarily chosen values of non-resonant frequency detuning $\delta=\lambda, 2 \lambda, 3 \lambda, 4 \lambda$ and photon number $n=0,1,2$ in Figs. 2 - 5. We note in Figs. 2 - 5 that the dynamical behaviour of quantum entanglement measured by concurrence is in the range $0 \leq C(\tau) \leq 1$, such that $\min C(\tau)=0$ and $\max C(\tau)=1$.

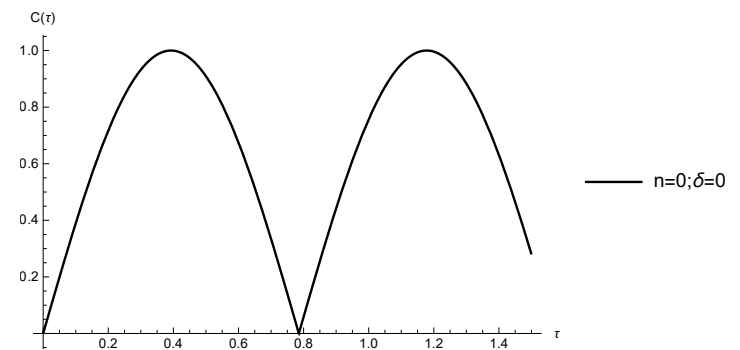

FIG. 2: Concurrence $C(\tau)$ against scaled time $\tau$ at resonance $\delta=0$ when $n=0$.

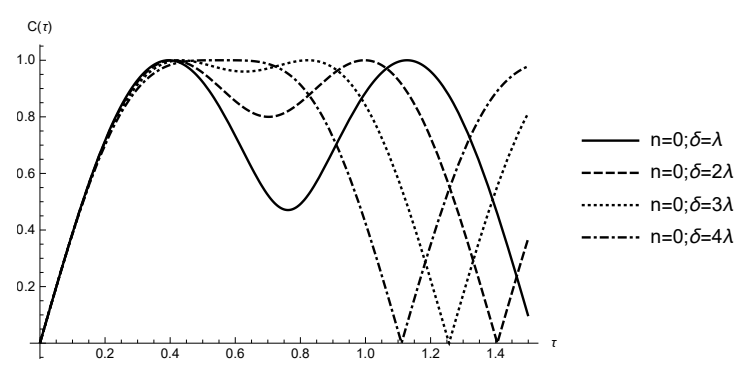

FIG. 3: Concurrence $C(\tau)$ against scaled time $\tau$ at offresonance $\delta=\lambda, \delta=2 \lambda, \delta=3 \lambda$ and $\delta=4 \lambda$ when $n=0$.

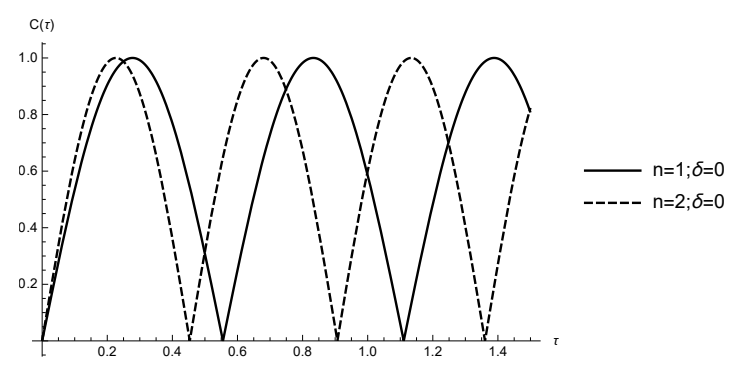

FIG. 4: Concurrence $C(\tau)$ against scaled time $\tau$ at resonance $\delta=0$ when $n=1,2$.

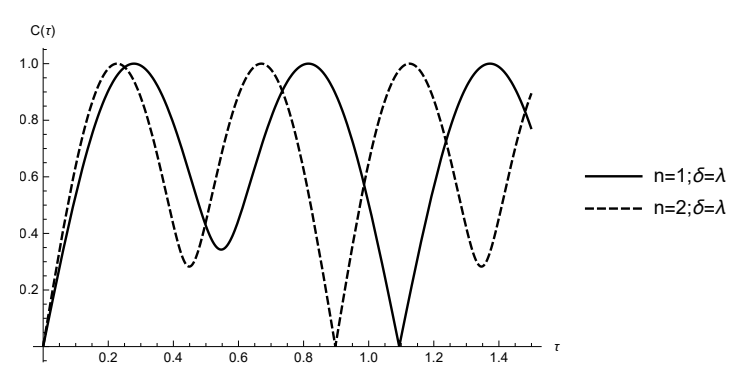

FIG. 5: Concurrence $C(\tau)$ against scaled time $\tau$ at offresonance $\delta=\lambda$ when $n=1,2$.

Comparing Fig. 2 and Fig. 3 we observe that at resonance $\delta=0$ the frequency of oscillation of $C(\tau)$ as shown in Fig. 2 is higher than when detuning is set at off resonance $\delta \neq 0$ as demonstrated in Fig. 3 for the specific 
cases of $\delta=\lambda, 2 \lambda, 3 \lambda, 4 \lambda$. In addition, Figs. 2 and 3 clearly show that there are Rabi oscillations even for the case when $n=0$, commonly referred to as vacuum-field Rabi oscillations [1, 24, 25]. Further we observe in Fig. 3, that despite the reduction in frequency of oscillation of $C(\tau)$ when $\delta \neq 0$ in comparison to the resonance case $\delta=0$ in Fig. 2, the frequency of oscillation of $C(\tau)$ increases with an increase in detuning $\delta \neq 0$. In the specific case of an initial photon number $n=0$ and frequency detuning $\delta=4 \lambda$ as demonstrated in Fig. 3, we observe a long-lived entanglement at $C(\tau)=1$. This agrees precisely with the result presented in Sec. IV A, where the transition qubit state vector obtained for parameter values $n=0$ and $\delta=4 \lambda$ was determined to be maximally entangled (resulting into the long-lived entanglement) before returning momentarily to a separable state. What is more, with reference to Fig. 3 it is evident that there is gradual vanishing of local minimums with increasing frequency detuning $\delta \neq 0$ indicating enhancement of entanglement.

To investigate the effect of photon number on the dynamics of $C(\tau)$ we considered a resonance case $\delta=0$ and varied the photon number $n$ in Fig. 4, while in Fig. 5, we set detuning constant at $\delta=\lambda$ and varied the photon number $n$. In both cases, we observe that the frequency of oscillation of $C(\tau)$ increases with an increase in photon number $n$.

Finally in all the plots in Figs. 2 - 5, entanglement sudden birth (ESB) and entanglement sudden death (ESD) is observed during time evolution of $C(\tau)$. In ESB, there is an observed creation of entanglement where the initially un-entangled qubits are entangled after a very short time interval. Consequently DEM decreases and goes to zero over a short period of time, where the system returns momentarily to a separable state. These findings in Sec. IV B are consistent with those obtained in [26-28] as examples.

\section{CONCLUSION}

We have analysed entanglement of a two-level atom and a single mode quantized electromagnetic field in a $\mathrm{JC}$ qubit formed in the JC interaction mechanism. The results confirm that in a resonant JC interaction, Rabi state transition oscillation occur between an initial and transition pure separable qubit states while in a nonresonant JC interaction Rabi state transition oscillations occur between an initial pure separable qubit state and a pure entangled transition qubit state where the degree of entanglement varies from entangled to maximally entangled depending on the set frequency detuning.

The effect of detuning parameter and photon number on the dynamical behaviour of entanglement in both resonant and non-resonant atom-field JC interaction measured by concurrence was studied giving a result similar to that obtained in the analysis of quantum state configurations on the Bloch sphere. The findings presented features such as long-lived entanglement, local minimums, entanglement sudden birth and entanglement sudden death.

In general, the results obtained in this work confirm that frequency detuning and photon number as parameters in JC interaction are fundamental in enhancing entanglement which plays a central role in quantum information and computation consistent with previous studies of the JC model cited herein.

\section{ACKNOWLEDGMENT}

We thank Maseno University Department of Physics and Materials Science for providing a conducive environment to do this work.

\section{CONFLICTS OF INTEREST}

The authors declare no conflicts of interest regarding the publication of this paper.
[1] E. T. Jaynes and F. W. Cummings, Proceedings of the IEEE 51, 89 (1963).

[2] I. Rabi, Phys. Rev. 49, 324 (1936).

[3] I. I. Rabi, Phys. Rev. 51, 652 (1937).

[4] J. A. Omolo, arXiv preprint arXiv:2103.06577 (2021).

[5] J. A. Omolo, preprint Research Gate, DOI:10.13140/RG.2.2.11833.67683 (2017).

[6] J. A. Omolo, preprint Research Gate, DOI: 10.13140/RG.2.2.27331.96807 (2019).

[7] J. Kukliński and J. Madajczyk, Phys. Rev. A 37, 3175 (1988).

[8] J. Gea-Banacloche, Phys. Rev. A 47, 2221 (1993).

[9] A. Kundu, Theor. Math. Phys. 144, 975 (2005).
[10] V. Hussin and L. Nieto, J. Math. Phys. 46, 122102 (2005).

[11] P. Lougovski, F. Casagrande, A. Lulli, and E. Solano, Phys. Rev. A 76, 033802 (2007).

[12] M. Bina, F. Casagrande, A. Lulli, and E. Solano, Phys. Rev. A 77, 033839 (2008).

[13] Q. Xie, H. Zhong, M. T. Batchelor, and C. Lee, J. Phys. A Math. Theor. 50, 113001 (2017).

[14] C. Mayero, J. A. Omolo, and O. S. Okeyo, Journal of Modern Physics 12, 408 (2021).

[15] M. Boyer, R. Liss, and T. Mor, Phys. Rev. A 95, 032308 (2017).

[16] B. Regula and G. Adesso, Phys. Rev. Lett. 116, 070504 (2016). 
[17] M. A. Nielsen and I. L. Chuang, Quantum Computation and Quantum Information (Cambridge University Press, 2011).

[18] E. G. Rieffel and W. H. Polak, Quantum computing: A gentle introduction (MIT Press, 2011).

[19] R. Van Meter, Quantum networking (John Wiley \& Sons, 2014).

[20] M. Enríquez, R. Reyes, and O. Rosas-Ortiz, J. Phys. Conf. Ser. 512, 012021 (2014).

[21] W. K. Wootters, Phys. Rev. Lett. 80, 2245 (1998).

[22] G. Jaeger, Entanglement, information, and the interpretation of quantum mechanics (Springer Science \& Business Media, 2009).

[23] S. Hill and W. K. Wootters, Phys. Rev. Lett. 78, 5022 (1997).
[24] R. Paschotta et al., Encyclopedia of laser physics and technology, vol. 1 (Wiley Online Library, 2008).

[25] T. Yoshie, A. Scherer, J. Hendrickson, G. Khitrova, H. Gibbs, G. Rupper, C. Ell, O. Shchekin, and D. Deppe, Nature 432, 200 (2004).

[26] S.-Q. Zhang, J.-B. Lu, X.-J. Liu, Y. Liang, H. Li, J. Ma, J.-P. Liu, and X.-Y. Wu, Int. J. Theor. Phys. 57, 279 (2018).

[27] S. Abdel-Khalek, M. Quthami, and M. Ahmed, Opt. Rev. 22, 25 (2015).

[28] M. Mohammadi and S. Jami, Optik 181, 582 (2019). 